\title{
Tetralogy of Fallot: cardiac imaging evaluation
}

\author{
Carlos Jerjes Sánchez Ramírez, Leopoldo Pérez de Isla \\ Department of Cardiac Imaging, Hospital Clínico San Carlos, Professor Martín Lagos, Madrid, Spain \\ Contributions: (I) Conception and design: All authors; (II) Administrative support: None; (III) Provision of study materials or patients: None; (IV) \\ Collection and assembly of data: CJ Sánchez Ramírez; (V) Data analysis and interpretation: All authors; (VI) Manuscript writing: All authors; (VII) \\ Final approval of the manuscript: All authors. \\ Correspondence to: Carlos Jerjes Sánchez Ramírez. Imaging Fellow at Department of Cardiac Imaging, Hospital Clínico San Carlos, Professor Martín \\ Lagos, s/n, 28040 Madrid, Spain. Email: carlosjerjes@gmail.com.
}

\begin{abstract}
Thanks to advances in pediatric cardiology, most infants with tetralogy of Fallot (TOF) now survive into adulthood. This relatively new population of adult patients may face long-term complications, including pulmonary regurgitation (PR), right ventricular (RV) tract obstruction, residual shunts, RV dysfunction, and arrythmias. They will often need to undergo pulmonary valve (PV) replacement and other invasive re-interventions. However, the optimal timing for these procedures is challenging, largely due to the complexity of evaluating RV volume and function. The options for the follow-up of these patients have rapidly evolved from an angiography-based approach to the surge of advanced imaging techniques, mainly echocardiography, cardiac magnetic resonance (CMR), and computer tomography (CT). In this review, we outline the indications, strengths and limitations of these modalities in the adult TOF population.
\end{abstract}

Keywords: Computed tomography; echocardiography; cardiac magnetic resonance (CMR); tetralogy of Fallot (TOF)

Submitted Dec 15, 2019. Accepted for publication Jan 20, 2020.

doi: $10.21037 /$ atm.2020.02.18

View this article at: http://dx.doi.org/10.21037/atm.2020.02.18

\section{Introduction}

Tetralogy of Fallot (TOF) is the most common cyanotic congenital heart disease (CHD); its prevalence is estimated at around 5/10,000 births (1). Furthermore, due to the success of pediatric cardiac surgery and pediatric cardiology, the population of adults with TOF is growing steadily, with over $90 \%$ of children with CHD now surviving into adulthood (2). This relatively new population of adult patients, which now outnumbers the pediatric TOF population, poses further challenges for clinicians. Cardiac imaging has rapidly evolved, from relying exclusively on cardiac catheterization and chest radiography (Figure 1) to today's more advanced imaging techniques: echocardiography, cardiac magnetic resonance (CMR), and cardiovascular computer tomography (CT) (3). Because of the complex nature and diversity of these population, as well as the limitations of each modality, it is increasingly recognized that an expert multidisciplinary team with a multimodality imaging approach is necessary for their clinical management (4).

\section{Preliminary assessment}

The adult TOF population is heterogeneous. Each patient is highly unique depending on several factors which will lead the imaging technique for their evaluation.

While TOF is classically defined by four hallmark findings, many patients exhibit a range of associated defects, including branch pulmonary stenosis (PS, in $40 \%$ of cases), right aortic arch $(25 \%)$, ascending aortic dilatation with or without aortic regurgitation (15\%), atrial septal defect (10\% - called 'pentalogy of Cantrell'), and anomalous coronary origin (8\%), among others (5) .

Furthermore, there is great variation in the status of previous interventions, which determines the expected complications. Most patients have undergone complete repair, and eventually may present the expected late 


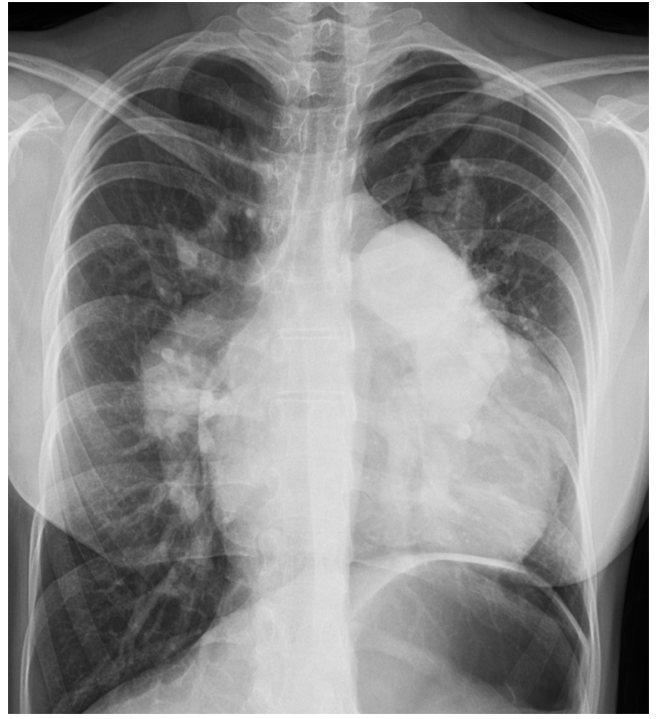

Figure 1 Chest X-ray of a 26-year-old patient with history of complete TOF repair in infancy. Note the severe cardiomegaly and RVOT dilatation. TOF, tetralogy of Fallot; RVOT, right ventricular outflow tract.

pulmonary regurgitation (PR) leading to right ventricular (RV) dilatation and dysfunction (6). In older patients, staged repairs were more common, and occasionally the adult patient may present to consultation with only the initial palliative shunt, such as the Waterson, Potts or BlalockTaussig procedures; in time, these may become obstructed or deteriorated. It is also more common for older patients to have had extensive trans-annular patching at the pulmonary valve (PV), while younger patients are more likely to have been treated with an infundibulum-sparing approach, which may reduce late RV dilation and dysfunction (7). And rarely, adult patients may have had neither palliative nor repair procedures, particularly in low income countries or with no access to health care; these patients have the worst prognosis. Finally, as the survival of repaired TOF patients increases, some of them will eventually develop acquired heart disease (8), and may require functional assessment of ischemia or imaging of their coronary arteries. Hence, a comprehensive understanding of a patient's medical and surgical record is necessary to guide individualized diagnostic imaging (4).

\section{Echocardiography}

The transthoracic echocardiogram (TTE) is the initial and main imaging technique in TOF, both pre- and post-
Table 1 Echocardiographic assessment of repaired TOF $(4,8,9)$

Residual RVOT obstruction; aneurysm

Main and branch PAs diameters

RVOT and Right ventricle to PA conduits

Native or prosthetic pulmonary regurgitation

Residual valvular or supravalvular pulmonary stenosis

$\mathrm{RV}$ volume, pressure, hypertrophy, and function

LV size and function

Tricuspid regurgitation

Residual VSD and ASD

Aortic root and ascending aortic dimensions

Aortic regurgitation

Systemic-to-pulmonary collateral vessels

TOF, tetralogy of Fallot; RVOT, right ventricular outflow tract; $\mathrm{PA}$, pulmonary artery; RV, right ventricle; VSD, ventricular septal defect; ASD, atrial septal defect.

operatively (9). It is the first-line modality throughout the patients' lifetimes; from fetal diagnosis to evaluation of late complications in older adults. TTE has the advantage of being inexpensive, universally available, and with no known biologic adverse effects. Standard clinical practice is to guide initial clinical management with TTE; in many patients it may even be the only imaging technique needed for follow-up. Annual TTE should be performed in patients with significant PR or aortic root dilatation; asymptomatic patients with an uncomplicated evolution may be followed less frequently, every 2 years (10). However, the echocardiographic examination in these patients requires advanced training and should be methodical and comprehensive, as summarized in Table 1.

Current guidelines $(4,8)$ recommend a standardized echocardiographic protocol in TOF patients. The precise evaluation of $\mathrm{PR}$ - a universal finding - and the main driver of RV dilatation and dysfunction, is essential. Theoretically, PR may be quantified with the same methods as aortic regurgitation. Proposed values for severe regurgitation include an effective regurgitant orifice area (EROA) greater than $115 \mathrm{~mm}^{2}$, regurgitant volume $>115 \mathrm{~mL}$, pressure half time $<100 \mathrm{~ms}$, a PR index $<0.77$, jet width $>65 \%$ of the right ventricular outflow tract (RVOT) width, and diastolic flow reversal in PA branches (Figure 2) (11); however, these values are not robust and in clinical practice there is no 


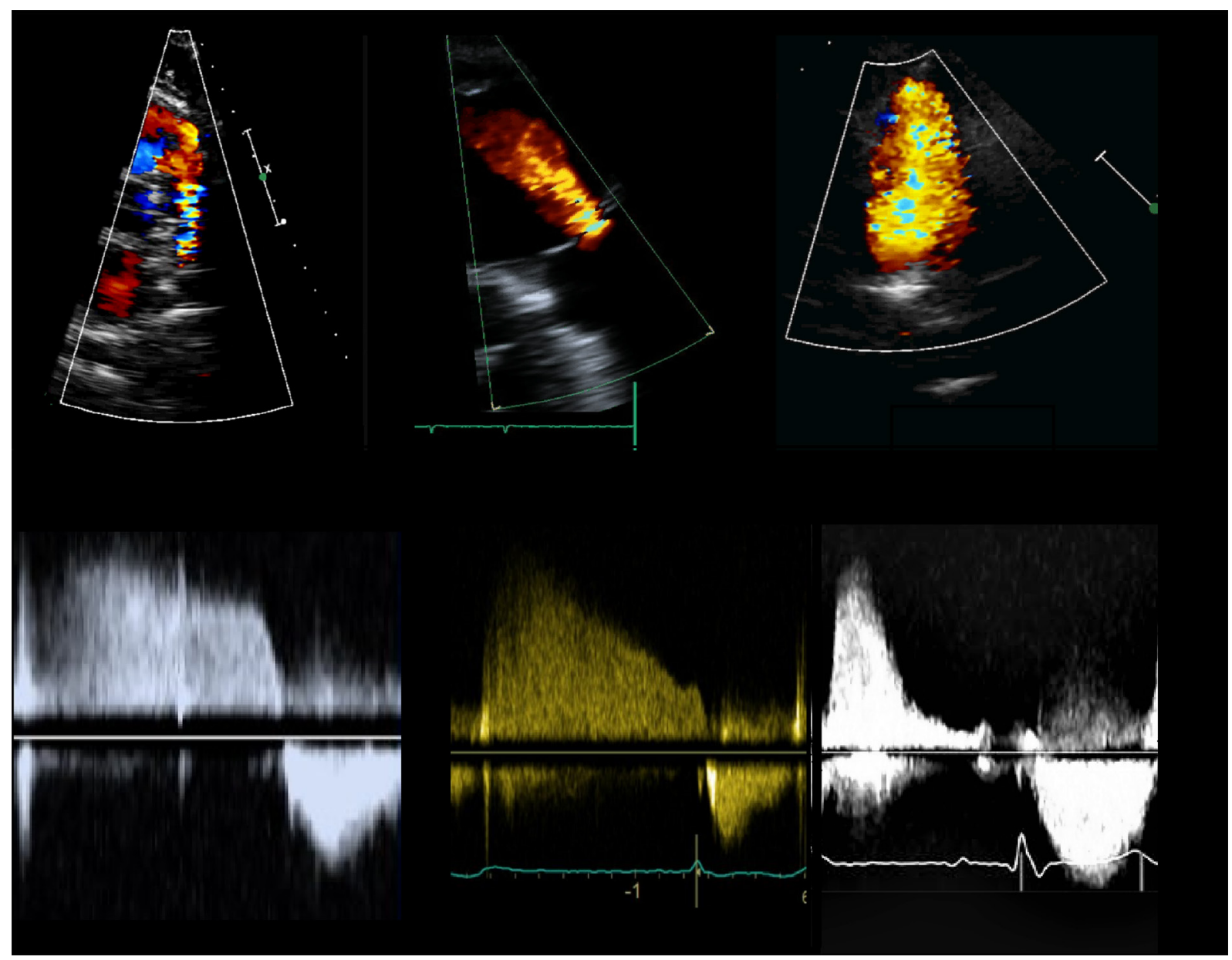

Figure 2 Different degrees of PR after TOF complete repair. Left: Mild PR. Center: Moderate PR. Right: Severe PR. Note the wide jet and steep deceleration of the continuous wave doppler curve compared to the mild PR curve. TOF, tetralogy of Fallot; PR, pulmonary regurgitation.

widespread accepted method for quantifying PR with TTE. Furthermore, RV dilatation, the functional consequence of $\mathrm{PR}$, is sensible but not specific for severe PR (12). For these reasons, grading PR severity by TTE remains challenging. Furthermore, while PR is the most common valvulopathy, other frequent findings are functional tricuspid regurgitation, aortic regurgitation, and residual pulmonary or subpulmonary stenosis.

Regarding RV function, the prognostic value of standard 2D TTE measurements in TOF patients is limited by paucity of data. Tricuspid annular plane systolic excursion (TAPSE), values have been shown to be lower in TOF and to become progressively reduced after surgery compared to age-matched controls (13). While 2D TTE 'normal' thresholds in TOF are difficult to determine, TAPSE, fractional area change (FAC), and tissue Doppler S' velocity are recommended by guidelines during follow-up $(4,8)$. However, it is also recognized that these parameters alone weakly correlate with CMR values of RV function $(13,14)$.

In most patients 2D TTE is insufficient for effectively evaluating the complex, nonstandard, retrosternal shape and function of the RV. 3D echocardiography overcomes this limitation and is accurate and reproducible for assessment of both RV volumes and function; furthermore, it is feasible in up to $90 \%$ of patients, with high correlation to CMR values $(15,16)$. Hence, 3D echocardiography RV volumes and EF are now preferred over 2D measurements (8). However, this technique requires software and equipment that aren't widely available (15).

There has also been recent interest in non-geometric 
Table 2 CMR protocol in repaired TOF $(4,8,12)$

Anatomical sequences
Right ventricle and RVOT morphology
Aortic override severity
Aortic arch
Cine sequences
Biventricular volumes and function
Presence of Pulmonary regurgitation
Presence of RVOT aneurysm
Phase contrast sequences
Pulmonary regurgitation
Aortic regurgitation
Pulmonary-to-systemic blood flow
Angio-MR
Great vessels
Pulmonary artery stenosis
Lurgical fistulas
Replacement fibrosis

CMR, cardiac magnetic resonance; TOF, tetralogy of Fallot; RVOT, right ventricular outflow tract.

indices of RV function, such as the myocardial performance index (17), electromechanical dyssynchrony, (18) and myocardial deformation, with limited clinical applicability. $\mathrm{RV}$ strain is a reliable method for evaluating subclinical myocardial damage and function. Previous studies have shown that RV strain deteriorates postoperatively but later recovers (19), with possible long-term deterioration (20). Whether this information is clinically relevant is still to be determined.

\section{Limitations of the technique}

The pediatric assessment in repaired TOF patients can usually be done entirely by TTE thanks to their excellent acoustic windows. Unfortunately, as patients age and transition into adolescence and adult age, image resolution tends to worsen (21). Artifacts created by previous chest surgery may also contribute to poor acoustic windows. Additionally, accurate evaluation of RV size and function is often not possible, even with 3D assessment (15). Hence,

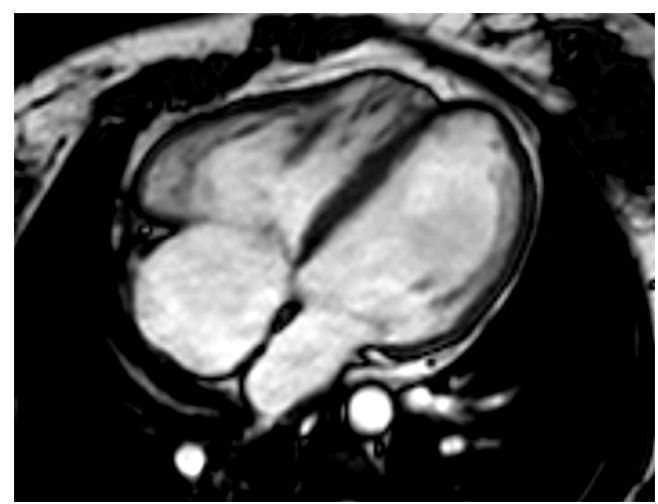

Figure 3 Cine CMR 4 chamber view of a patient with repaired TOF and positive clinical evolution. The RV is mildly dilated and with mild RV hypertrophy. CMR, cardiac magnetic resonance; TOF, tetralogy of Fallot; RV, right ventricular.

CMR remains the preferred method for RV quantitative assessment in most patients (3).

\section{Magnetic resonance}

$\mathrm{CMR}$ is fundamental for the surveillance in the adult patient with CHD. While TTE can provide most of the information necessary for serial follow-up in TOF, CMR is indicated in several scenarios: When TTE is of suboptimal quality, when there is discrepancy between TTE and clinical evaluation, for tissue characterization, and as an alternative to invasive catheterization (21). CMR is usually not needed during the first decade of life, as TTE is diagnostic in most patients (8). The exception are the patients with pulmonary atresia and aortopulmonary collaterals, who do need additional imaging for characterization. The value of CMR increases at adolescence. In stable, asymptomatic TOF patients, baseline CMR should be performed at the transition from the pediatric to the ACHD programs; afterwards it should be indicated routinely every 3 years. On patients with more advanced, complicated disease, CMR followup interval should be performed every 1 to 2 years (10). The goals of CMR in TOF are summarized in Table 2.

In clinical practice, CMR's principal advantage over TTE is the accurate quantification of biventricular size and function, and it is the gold standard for volume and flow assessment in TOF (12) (Figure 3). Optimal timing for PV replacement in PR is challenging, and current recommendations rely on $\mathrm{RV}$ volumes, which are performed with cine steady-state free precession (SSFP) 


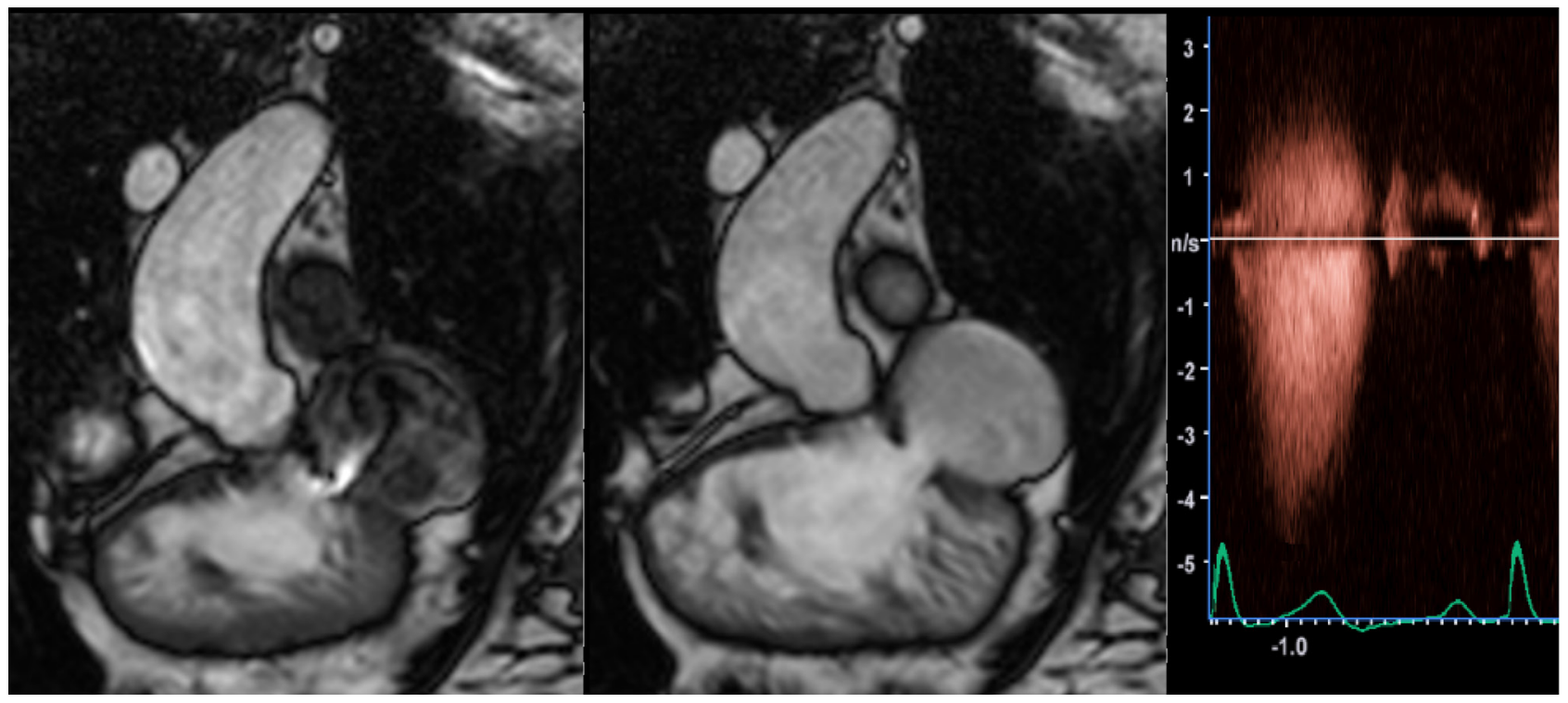

Figure 4 CMR cine images depicting a patient with PS in systole (left) and diastole (center). The doppler curve (right), with a maximum velocity close to $5 \mathrm{~m} / \mathrm{s}$, resembles an aortic stenosis curve, and corresponds to severe PS. CMR, cardiac magnetic resonance; PS, pulmonary stenosis.

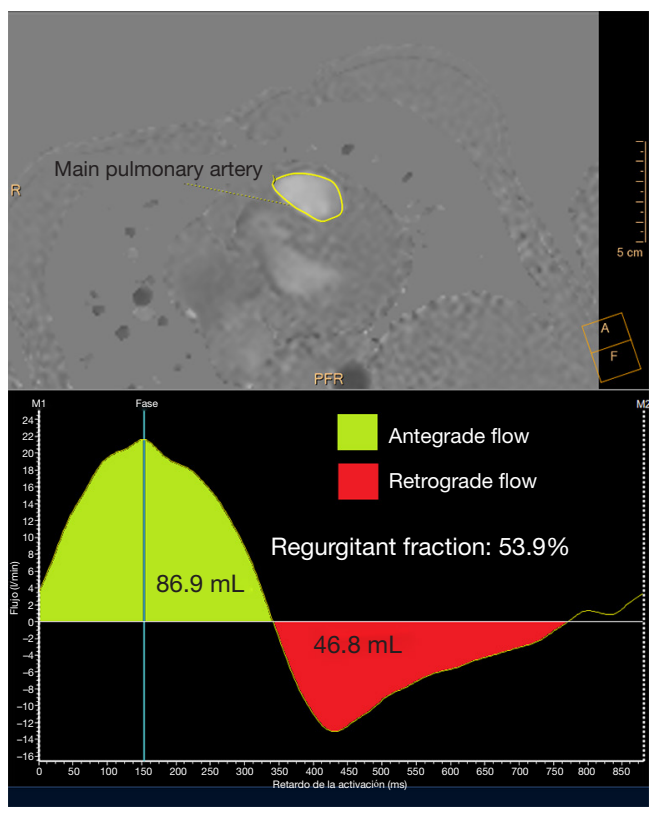

Figure 5 Regurgitant fraction calculation in a patient with repaired TOF and severe PR. The first image displays the imaging plane, perpendicular to the proximal main pulmonary artery. The second image shows volume and direction of flow over time. TOF, tetralogy of Fallot; PR, pulmonary regurgitation. sequences. Generally, PV replacement should be indicated before reaching an RV end-diastolic indexed volume of $160 \mathrm{~mL} / \mathrm{m}^{2}$ or an $\mathrm{RV}$ end-systolic indexed volume of $80 \mathrm{~mL} / \mathrm{m}^{2}$ (10,22). These are CMR thresholds considered a point of no return beyond which RV function and reverse remodeling recovery would be expected to be limited after PV replacement $(23,24)$. On the other hand, intervening too early potentially exposes patients to reinterventions later in life. Consequently, since the indication for PV replacement depends on CMR, it should be indicated in all patients prior to deciding intervention. Cine sequences also allows us to assess RVOT wall motion and geometry, including obstruction to flow, detect valvular regurgitation and stenosis (Figure 4) and residual shunts, and evaluate aortic dilatation.

CMR is the gold standard for blood flow measurements, particularly quantification of regurgitant volume in PR. The phase contrast sequence provides measurement of anterograde and retrograde flow to establish regurgitant fraction and establish PR severity (8) (Figure 5). A regurgitant fraction $>40 \%$ is considered severe. Phase contrast also permits assessment of aortic regurgitation, and in patients with residual shunts, estimation of pulmonary- 


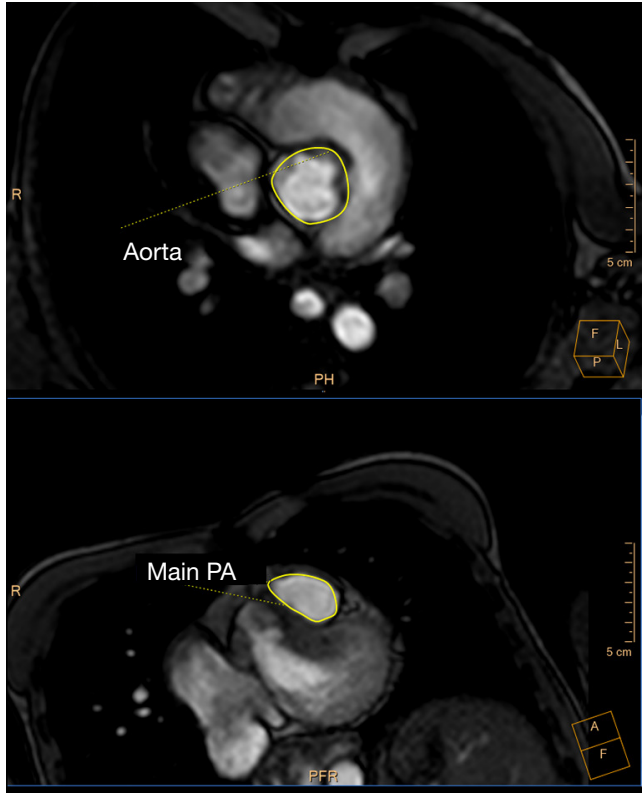

Figure 6 Imaging planes for calculation of pulmonary-to-systemic blood flow calculation (Qp:Qs)

to-systemic blood flow (Qp:Qs) (25) (Figure 6) However, aortic or tricuspid regurgitation, common in TOF, limits the use of this technique.

Contrast-enhanced CMR angiography is ideal for assessing the arterial and venous systems, particularly the pulmonary arteries for residual stenosis. In these cases, differential PA branch flow with phase-contrast measurement aids in determining intervention. RVOT aneurysms, the permeability of surgical fistulas, coronary artery anomalies, and the aortic root and ascending aorta are also evaluated with this technique (4).

Finally, CMR can characterize cardiac tissue. Late gadolinium enhancement detects focal fibrosis and is a marker for risk of RV dysfunction, ventricular arrythmias and sudden cardiac death after TOF repair (26). More recently, increased extracellular volume calculated with pre- and post-gadolinium T1 measurements has also been associated with RV volume overload and arrhythmias, suggesting measurements of diffuse fibrosis may help stratify patient risk (27).

\section{Limitations of the technique}

Its principal disadvantage is higher cost compared with TTE. Limited availability is offset in developed countries, where TOF patients are usually followed in

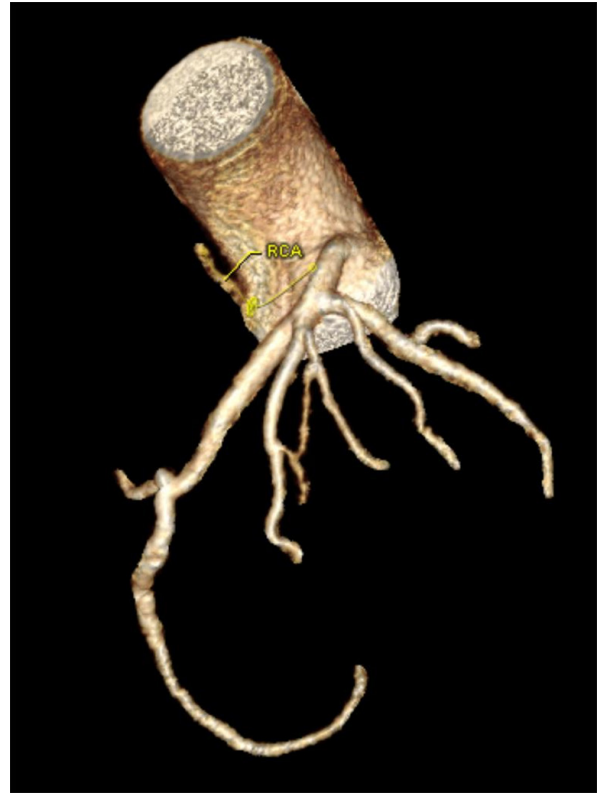

Figure 7 CT reconstruction of a patient with an absent right coronary artery.

tertiary centers. While CMR quality is not affected by acoustic windows, there may be artifacts arising from steel implants. Additionally, some patients may carry noncompatible pacemakers or defibrillators, in whom CMR is contraindicated. Finally, there is a small risk of nephrogenic systemic fibrosis with gadolinium contrast in patients with advanced renal disease (14).

\section{Computer tomography}

While CMR in TOF is performed much more often than $\mathrm{CT}$, the former is superior in several aspects. Its main advantage is that multidetector CT (MDCT) has the highest spatial resolution of all imaging techniques (3). This allows a more detailed assessment of small vessel anatomy, including pulmonary veins, distal pulmonary artery branches, and aortopulmonary collaterals (25).

It is also useful for coronary artery imaging, since an anomalous coronary origin occurs in $8-12 \%$ of TOF patients (Figure 7) (28). The most common finding is the left anterior descending artery arising from the right coronary artery and passing through the RVOT. This variation precludes surgical procedures on the RVOT. Coronary CT may also exclude significant ischemic heart disease in selected cases.

In practice, MDCT in TOF is commonly indicated in 
the older patient with planned reinterventions in whom it may substitute invasive angiography. In these patients MDCT permits assessment of calcification burden in surgical conduits (unlike CMR), information which might be useful for the interventionist (8). MDCT is also the imaging modality of choice for serial evaluation of stent patency, integrity, morphology, and position (29).

Finally, CT is being used for accurate measurement of the RVOT before transcatheter PV implantation (30). Finally, CT is the imaging technique of choice for anatomic information in patients with contraindications to cardiac MRI, though functional information comes at the expense of retrospective gating, with higher radiation dose (12).

\section{Limitations of the technique}

Its chief limitation is that CT exposes the patient to ionizing radiation, which increases the risk of cancer (31). This is inconvenient in a relatively young population which will need repeat exposures. It also is unable to provide information on blood flow, unlike CMR and TTE. These modalities also have higher temporal resolution than CT (14). Finally, contrast agents also carry a risk of renal injury in some patients (8).

\section{Conclusions-multimodality imaging}

The previous discussion highlights the large spectrum of severity and diverse imaging goals in TOF patients. While the three major imaging techniques have strengths and limitations, none is adequate for a comprehensive approach to these patients. Therefore, a multimodality approach considering each patient's specific needs should be sought. Echocardiography is the first and most frequently used technique for serial evaluation in most patients. In young patients, with good windows and few expected complications, it may be the only test needed. However, as patients age, multiple complications may arise, and in many cases the echocardiogram will be insufficient for guiding management. Specific issues where CMR becomes important are the timing of $\mathrm{PV}$ replacement, accurate blood flow assessment, tissue characterization, and evaluation of extra-cardiac and great vessel anatomy. On the other hand, MDCT is important as a substitute for invasive angiography, when more detailed assessment of cardiac and small vessel anatomy is needed, as with the coronaries, and for assessment of stents and conduits. It is also an alternative for patients in whom CMR is contraindicated or inconvenient. In summary, echocardiography should be routine for every patient, CMR should be used regularly in patients with complications, and MDCT in selected patients to answer specific questions.

\section{Acknowledgments}

Funding: None.

\section{Footnote}

Provenance and Peer Review: This article was commissioned by the Guest Editor (Daniel Hernández-Vaquero) for the series "Structural Heart Disease: The Revolution" published in Annals of Translational Medicine. The article was sent for external peer review organized by the Guest Editor and the editorial office.

Conflicts of Interest: Both authors have completed the ICMJE uniform disclosure form (available at http://dx.doi. org/10.21037/atm.2020.02.18). The series "Structural Heart Disease: The Revolution" was commissioned by the editorial office without any funding or sponsorship. The authors have no other conflicts of interest to declare.

Ethical Statement: The authors are accountable for all aspects of the work in ensuring that questions related to the accuracy or integrity of any part of the work are appropriately investigated and resolved.

Open Access Statement: This is an Open Access article distributed in accordance with the Creative Commons Attribution-NonCommercial-NoDerivs 4.0 International License (CC BY-NC-ND 4.0), which permits the noncommercial replication and distribution of the article with the strict proviso that no changes or edits are made and the original work is properly cited (including links to both the formal publication through the relevant DOI and the license). See: https://creativecommons.org/licenses/by-nc-nd/4.0/.

\section{References}

1. Reller MD, Strickland MJ, Riehle-Colarusso T, et al. Prevalence of Congenital Heart Defects in Metropolitan Atlanta, 1998-2005. J Pediatr 2008;153:807-13.

2. Mulder BJM. Epidemiology of adult congenital heart disease: demographic variations worldwide. Neth Heart J 
2012;20:505-8.

3. Han BK, Lesser AM, Vezmar M, et al. Cardiovascular imaging trends in congenital heart disease: A single center experience. J Cardiovasc Comput Tomogr 2013;7:361-6.

4. Valente AM, Cook S, Festa P, et al. Multimodality Imaging Guidelines for Patients with Repaired Tetralogy of Fallot: A Report from the American Society of Echocardiography. J Am Soc Echocardiogr 2014;27:111-41.

5. Kochav, J. Adult congenital heart disease in clinical practice. In: DeFariah Yeh D, Bhatt A. Tetralogy of Fallot New York, NY: Springer Berlin Heidelberg; 2018. Chapter 23:295-318.

6. Mosca RS. Pulmonary valve replacement after repair of tetralogy of Fallot: Evolving strategies. J Thorac Cardiovasc Surg 2016;151:623-5.

7. Bové T, François K, Van De Kerckhove K, et al. Assessment of a right-ventricular infundibulumsparing approach in transatrial-transpulmonary repair of tetralogy of Fallot. Eur J Cardiothorac Surg 2012;41:126-33.

8. Di Salvo G, Miller O, Babu Narayan S, et al. Imaging the adult with congenital heart disease: a multimodality imaging approach-position paper from the EACVI. Eur Heart J Cardiovasc Imaging 2018;19:1077-98.

9. DeFaria Yeh D, King ME. Congenital Heart Disease in the Adult: What Should the Adult Cardiologist Know? Curr Cardiol Rep 2015;17:25.

10. Stout KK, Daniels CJ, Aboulhosn JA, et al. 2018 AHA/ACC Guideline for the Management of Adults With Congenital Heart Disease. J Am Coll Cardiol 2019;73:e81-192.

11. Lancellotti P, Tribouilloy C, Hagendorff A, et al. European Association of Echocardiography recommendations for the assessment of valvular regurgitation. Part 1: aortic and pulmonary regurgitation (native valve disease). Eur J Echocardiogr 2010;11:223-44.

12. Kochav J, Simprini L, Weinsaft JW. Imaging of the Right Heart-CT and CMR. Echocardiography 2015;32:S53-68.

13. Koestenberger M, Nagel B, Ravekes W, et al. Tricuspid annular plane systolic excursion and right ventricular ejection fraction in pediatric and adolescent patients with tetralogy of Fallot, patients with atrial septal defect, and age-matched normal subjects. Clin Res Cardiol 2011;100:67-75

14. DeFaria Yeh D, Foster E. Is MRI the Preferred Method for Evaluating Right Ventricular Size and Function in
Patients With Congenital Heart Disease?: MRI Is Not the Preferred Method for Evaluating Right Ventricular Size and Function in Patients With Congenital Heart Disease. Circ Cardiovasc Imaging 2014;7:198-205.

15. Dragulescu A, Grosse-Wortmann L, Fackoury C, et al. Echocardiographic assessment of right ventricular volumes: a comparison of different techniques in children after surgical repair of tetralogy of Fallot. Eur Heart J Cardiovasc Imaging 2012;13:596-604.

16. Dragulescu A, Grosse-Wortmann L, Fackoury C, et al. Echocardiographic Assessment of Right Ventricular Volumes after Surgical Repair of Tetralogy of Fallot: Clinical Validation of a New Echocardiographic Method. J Am Soc Echocardiogr 2011;24:1191-8.

17. Pavlicek M, Wahl A, Rutz T, et al. Right ventricular systolic function assessment: rank of echocardiographic methods vs. cardiac magnetic resonance imaging. Eur J Echocardiogr 2011;12:871-80.

18. Hui W, Slorach C, Dragulescu A, et al. Mechanisms of Right Ventricular Electromechanical Dyssynchrony and Mechanical Inefficiency in Children After Repair of Tetralogy of Fallot. Circ Cardiovasc Imaging 2014;7:610-8.

19. DiLorenzo MP, Elci OU, Wang Y, et al. Longitudinal Changes in Right Ventricular Function in Tetralogy of Fallot in the Initial Years after Surgical Repair. J Am Soc Echocardiogr 2018;31:816-21.

20. Li Y, Xie M, Wang X, et al. Impaired Right and Left Ventricular Function in Asymptomatic Children with Repaired Tetralogy of Fallot by Two-Dimensional Speckle Tracking Echocardiography Study. Echocardiography 2015;32:135-43.

21. Kutty S, Danford DA. Pediatric and Adult Congenital Heart Disease Imaging: Choices and Considerations. J Ultrasound Med 2013;32:1351-2.

22. Baumgartner H, Bonhoeffer P, De Groot N, et al. ESC Guidelines for the management of grown-up congenital heart disease (new version 2010): The Task Force on the Management of Grown-up Congenital Heart Disease of the European Society of Cardiology (ESC). Eur Heart J 2010;31:2915-57.

23. Bokma JP, Winter MM, Oosterhof T, et al. Preoperative thresholds for mid-to-late haemodynamic and clinical outcomes after pulmonary valve replacement in tetralogy of Fallot. Eur Heart J 2016;37:829-35.

24. Geva T, Gauvreau K, Powell AJ, et al. Randomized Trial of Pulmonary Valve Replacement With and Without Right Ventricular Remodeling Surgery. Circulation 
2010;122:S201-8.

25. Bonnichsen C, Ammash N. Choosing Between MRI and CT Imaging in the Adult with Congenital Heart Disease. Curr Cardiol Rep 2016;18:45.

26. Babu-Narayan SV, Kilner PJ, Li W, et al. Ventricular Fibrosis Suggested by Cardiovascular Magnetic Resonance in Adults With Repaired Tetralogy of Fallot and Its Relationship to Adverse Markers of Clinical Outcome. Circulation 2006;113:405-13.

27. Chen C-A, Dusenbery SM, Valente AM, et al. Myocardial ECV Fraction Assessed by CMR Is Associated With Type of Hemodynamic Load and Arrhythmia in Repaired Tetralogy of Fallot. JACC Cardiovasc Imaging 2016;9:1-10.

Cite this article as: Sánchez Ramírez CJ, Pérez de Isla L. Tetralogy of Fallot: cardiac imaging evaluation. Ann Transl Med 2020;8(15):966. doi: 10.21037/atm.2020.02.18
28. Kervancioglu M, Tokel K, Varan B, et al. Frequency, origins and courses of anomalous coronary arteries in 607 Turkish children with tetralogy of Fallot. Cardiol J 2011;18:546-51.

29. Ahmed S, Johnson PT, Fishman EK, et al. Role of Multidetector CT in Assessment of Repaired Tetralogy of Fallot. RadioGraphics 2013;33:1023-36.

30. Saremi F, Gera A, Yen Ho S, et al. CT and MR Imaging of the Pulmonary Valve. RadioGraphics 2014;34:51-71.

31. Lapierre C, Dubois J, Rypens F, et al. Tetralogy of Fallot: Preoperative assessment with MR and CT imaging. Diagn Interv Imaging 2016;97:531-41. 